\title{
The Effect of Plantar Massage on Static Postural Control in Patients With Chronic Ankle Instability: A Critically Appraised Topic
}

\author{
Katherine L. Helly, Katherine A. Bain, Phillip A. Gribble, and Matthew C. Hoch
}

\begin{abstract}
Clinical Scenario: Patients with chronic ankle instability (CAI) demonstrate deficits in both sensory and motor function, which can be objectively evaluated through static postural control testing. One intervention that has been suggested to improve somatosensation and, in turn, static postural control is plantar massage. Clinical Question: Does plantar massage improve static postural control during single-limb stance in patients with CAI relative to baseline? Summary of Key Findings: A search was performed for articles exploring the effect of plantar massage on static postural control in individuals with CAI. Three articles were included in this critically appraised topic including 1 randomized controlled trial and 2 crossover studies. All studies supported the use of plantar massage to improve static postural control in patients with CAI. Clinical Bottom Line: There is currently good-quality and consistent evidence that supports the use of plantar massage as an intervention that targets the somatosensory system to improve static postural control in patients with CAI. Future research should focus on incorporating plantar massage as a treatment intervention during long-term rehabilitation protocols for individuals with CAI. Strength of Recommendation: In agreement with the Center of Evidence-Based Medicine, the consistent results from 2 crossover studies and 1 randomized controlled trial designate that there is level B evidence due to consistent, moderate- to high-quality evidence.
\end{abstract}

Keywords: static balance, ankle injury, therapeutic massage

\section{Clinical Scenario}

Lateral ankle sprains are the most common musculoskeletal injury experienced by those who participate in physical activity, with approximately 23,000 people sustaining an ankle sprain each day in the United States. ${ }^{1}$ After completing supervised rehabilitation, $15 \%$ to $64 \%$ of patients who sprain their ankle do not report a full recovery within 3 years of their first sprain. ${ }^{2}$ Patients who develop lingering symptoms are more likely to have recurrent sprains and experience the feeling of "giving way." 3 Chronic ankle instability (CAI) is defined as having a history of at least 1 significant ankle sprain, a history of the previously injured ankle joint "giving way" and/or recurrent sprain and/or feelings of instability, and selfreported disability. ${ }^{4}$ Ultimately, CAI can significantly decrease physical function, health-related quality of life, and physical activity levels over the life span. ${ }^{5}$

Although patients with CAI exhibit many impairments, one of the most common long-term deficits is diminished static balance. ${ }^{6,7}$ Patients with CAI have also demonstrated reduced sensitivity on the plantar aspect of their foot, which may contribute to their balance deficits. ${ }^{8}$ Plantar massage is an intervention hypothesized to stimulate the plantar cutaneous receptors and increase tactile acuity. ${ }^{9}$ By augmenting sensory input, massage should improve the efficacy of motor output, and this sensorimotor alteration could be illustrated in the form of postural control measurements. Despite the potential benefits of plantar massage, few investigations have studied the isolated effects of this intervention on static postural control in individuals with CAI. Clinically, it is important to improve static postural control in hopes of potentially mitigating the self-reported symptoms associated with CAI while potentially

The authors are with the Department of Athletic Training and Clinical Nutrition, College of Health Sciences, University of Kentucky, Lexington, KY, USA. Hoch (matt.hoch@uky.edu) is corresponding author. improving these patients' health-related quality of life. Therefore, the aim of this critically appraised topic (CAT) was to identify, synthesize, and critically evaluate the evidence that has examined the effect of plantar massage on static postural control in patients with CAI. This will better assist health care professionals in applying evidence-based treatment strategies to address residual static balance impairments following an ankle sprain.

\section{Focused Clinical Question}

Does plantar massage improve static postural control during singlelimb stance in patients with CAI relative to baseline?

\section{Search Strategy}

A computerized search was performed in February 2020. The search terms used were:

- Patient/client group: CAI OR ankle instability

- Intervention/assessment: massage OR plantar massage

- Comparison: none (not included in search)

- Outcomes: static postural control OR center of pressure (COP) OR time to boundary

\section{Sources of Evidence Searched}

- CINAHL with full text

- MEDLINE

- SPORTDiscus

\section{Inclusion Criteria}

- Studies published in English

- Studies published in the last 10 years 
- Studies that used human subjects

- Studies that included patients with CAI

- Studies that examined outcomes following a plantar massage intervention

- Studies that objectively evaluated static postural control using a force plate

\section{Exclusion Criteria}

- Studies that examined healthy populations

- Studies that used balance training in conjunction with plantar massage

- Studies that used only subjective postural control outcomes

- Studies that were systematic reviews or meta-analyses

\section{Evidence Quality Assessment}

The Physiotherapy Evidence Database (PEDro) rating scale was used to systematically examine the research included to determine each study's methodological quality and usability for clinical decision making. ${ }^{10}$ The PEDro includes 10 criteria, therefore the maximum score an article can receive is 10 . The rating scale identifies both the trial's internal validity and statistical reporting. ${ }^{10}$ Two authors (K.L.H. and K.A.B.) independently reviewed and evaluated each study according to the PEDro scale. After the independent review, the 2 authors came to an agreement about the quality of each study. Studies scoring $\geq 60 \%$ on the PEDro were considered "moderate to high quality" evidence. ${ }^{11}$

\section{Results of Search}

\section{Summary of Search, Best Evidence Appraised, and Key Findings}

- A literature search was conducted in February 2020 to identify peer-reviewed articles that examined the use of plantar massage in participants with CAI and followed the inclusion and exclusion criteria listed earlier.

- The literature search yielded 7 articles. After review of the abstracts, 4 studies were excluded as they did not meet the inclusion criteria for this CAT (Figure 1). Two crossover studies $^{9,12}$ and 1 randomized controlled trial ${ }^{13}$ met the inclusion criteria. Study characteristics are listed in Table 1.

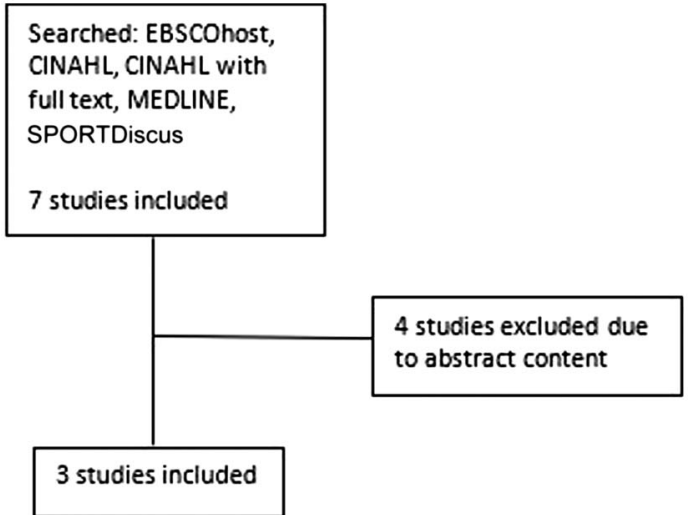

Figure 1 - Search strategy.
- All 3 studies ${ }^{9,12,13}$ measured COP during single-limb balance using a force plate. Wikstrom et al ${ }^{12}$ measured COP velocity and COP 95\% area ellipse during three 10-second test trials standing on the involved limb. LeClaire and Wikstrom ${ }^{9}$ measured COP excursion in the anterior-posterior (AP), mediallateral (ML), and the resultant directions as well as average COP sway in the the AP and ML directions. McKeon and Wikstrom ${ }^{13}$ measured COP-SD, COP velocity, and time to boundary in the AP and ML directions.

- Each study followed the same inclusion criteria for CAI following the guidelines set forth by the International Ankle Consortium. ${ }^{5}$ Participants must have had a history of at least 1 lateral ankle sprain, at least 2 episodes of giving way in the past 6 months, and score 5 or more on the Ankle Instability Instrument. In addition, McKeon and Wikstrom ${ }^{13}$ required participants to score less than $90 \%$ on the Foot and Ankle Ability Measure and less than $80 \%$ on the Foot and Ankle Ability Measure-Sport.

- Each study compared postural control measures before and after plantar massage. All 3 studies found improvements in postural control following plantar massage relative to baseline. ${ }^{9,12,13}$

\section{Results of Evidence Quality Assessment}

The PEDro was utilized on each included study (Table 1). Each study scored a 6/10. All studies lacked the same criteria, including the lack of blinding of the participants, clinicians delivering the intervention, or the assessors. In addition, none of the studies explicitly stated that they used concealed allocation when determining the group assignment or treatment sequences.

\section{Clinical Bottom Line}

There is currently moderate- to high-quality, consistent evidence that plantar massage improves static postural control in individuals with CAI relative to baseline measures. These improvements were identified immediately following treatment in all studies.9,12,13 In agreement with the Center of Evidence-Based Medicine, the consistent results from 2 crossover studies ${ }^{9,12}$ and 1 randomized controlled trial ${ }^{13}$ designate that there is level B evidence to support the use of plantar massage to improve static postural control in those with CAI.

\section{Implications for Practice, Education, and Future Research}

Our aim was to appraise the current evidence to determine whether plantar massage was an effective treatment for improving static postural control in patients with CAI. This CAT revealed moderateto high-quality and consistent evidence that plantar massage improved static postural control in individuals with CAI relative to baseline measures. The articles included in this CAT utilized different plantar massage interventions and treatment volumes in patients with CAI. Wikstrom et al ${ }^{12}$ identified statistically significant differences between COP velocity and COP 95\% area ellipse after each intervention session, with each session utilizing a different massage technique. Although this study found significant decreases in COP velocity and COP $95 \%$ area ellipse, it did not differentiate COP trajectories by direction. ${ }^{12}$ However, both clinician-delivered and patient-delivered massage improved outcome measures, indicating that there may not be a need for clinicians to 


\section{Table 1 Summary of Article Titles}

Title Comparative effectiveness ofplantar-massage techniques onpostural control in those with CAI

$\begin{array}{ll}\text { Authors } & \text { Wikstrom et al }{ }^{12} \\ \text { Participants } & \text { 20 physically active individuals (6 men and } \\ & \text { 14 women) with self-reported CAI } \\ & \text { Average age of participants: } 21.45 \mathrm{y}\end{array}$

Inclusion/ exclusion criteria $^{\mathrm{a}}$
Massage for postural control in individuals with CAI

LeClaire and Wikstrom ${ }^{9}$

18 volunteers with CAI from general student population at a large university Gender demographics not reported Average age of participants: $23.9 \mathrm{y}$

I: History of at least 1 ankle sprain, history of at least 2 episodes of "giving way" within past $6 \mathrm{mo}$, and answering yes to 5 or more questions on AII.

E: Sustaining an acute sprain within $6 \mathrm{wk}$, previous history of LE surgery, LE injury within the past 6 mo (other than ankle sprain), and neuropathy or other conditions known to affect balance.

\section{Study design Crossover study}

Methods

3 test sessions involving 3 treatments, a clinician-delivered plantar massage, a patient-delivered plantar massage, and a clinician-delivered sensory massage brush. A 7-d washout window was required in between treatments. Each session consisted on a pretest, treatment, and a posttest.

Three 10-s test trials with eyes open on single limb.

Testing

procedures

Outcome

measures

Static: COP-V, $95 \%$ area ellipse

Dynamic: reach distance \% (Ant, PM, PL)

Intervention Manual plantar massage

investigated Over the counter ball massager

Sensory brush

Results

Static postural control improved after each intervention.

No changes in SEBT

No differences between clinician-delivered interventions and self-administered

\begin{tabular}{lll}
$\begin{array}{l}\text { Appraisal } \\
\text { score }\end{array}$ & PEDro: 6/10 & PEDro: 6/10 \\
$\begin{array}{l}\text { Support of } \\
\text { answer }\end{array}$ & Yes & Yes \\
\hline
\end{tabular}
$\mathrm{R}-\mathrm{COP})$ occluded.

PEDro: 6/10 answer

\section{Crossover study}

Subjects were given treatment 2 times with at least a week in between. At the first test session, patients were baselined and given the first intervention, then balance tested immediately following. At the second test session, the same procedure occurred just with the other intervention.

Six 20-s test trials - 3 with eyes open, 3 with eyes closed-on single limb.

COP excursion (AP-COP, ML-COP,

COP sway (AP-sway, ML-sway)

5-min plantar massage

5-min calf massage

5 min of plantar massage immediately improved static postural control when vision was undisturbed. Neither intervention improved balance when vision was

PEDro: 6/10
The effect of sensory-targeted ankle rehabilitation strategies on single-leg center of pressure elements in those with CAI: a randomized control trial

McKeon and Wikstrom ${ }^{13}$

77 participants with self-reported CAI Gender demographics not reported Average age of participants not reports

I: Patients with self-reported CAI (defined as having a history of at least 2 episodes of "giving way" in $6 \mathrm{mo}$, greater the 5 score of AII, less than $90 \%$ on FAAM, and less than $80 \%$ on FAAM-S).

E: Failing to meet inclusion, sustaining an ankle sprain in the $6 \mathrm{wk}$ prior to screening, previous history of ankle surgery, lower extremity surgeries associated with internal derangements or repairs, and/or other conditions known to affect sensorimotor function.

Randomized control trial

Baseline measures were taken for COP data, patients were randomly allocated to 4 STARS groups (joint mobilizations, plantar massage, calf stretching, or control). Six 5min treatments over $2 \mathrm{wk}$.

Subjects were assessed 3 times, at baseline, immediately following the initial treatment, and within $72 \mathrm{~h}$ of completing the $2 \mathrm{wk}$ of treatment.

Six 10 -s test trials -3 with eyes open, 3 with eyes closed - on single limb.

COP-SD (ML COP-SD, AP COP-SD) COP-V (ML COP-V, AP COP-V)TTB (ML TTB, AP TTB)

Joint mobilizations

Plantar massage

Calf stretching

Control

Joint mobilizations produced immediate improvements in the temporal elements with eyes open.

Plantar massage enhanced temporal element after a single treatment with eyes closed.

Yes

Abbreviations: AII, Ankle Instability Instrument; Ant, anterior; AP, anterior-posterior; CAI, chronic ankle instability; COP, center of pressure; COP-V, COP velocity; FAAM, Foot and Ankle Ability Measure; FAAM-S, FAAM - Sport; LE, lower extremity; ML, medial-lateral; MSK, musculoskeletal; PEDro, Physiotherapy Evidence database rating scale; PL, posterolateral; PM, posteromedial; R-COP, resultant COP; SEBT, star excursion balance test; TTB, time to boundary.

${ }^{a}$ All studies follow the guidelines set forth by the International Ankle Consortium.

provide one-on-one massage interventions. ${ }^{12}$ LeClaire and Wikstrom $^{9}$ found statistically significant improvements in COP velocity and COP sway in both AP and ML directions with eyes open immediately after 5 minutes of plantar massage in patients with CAI. No significant differences were seen between static balance outcomes with eyes closed. Finally, McKeon and Wikstrom ${ }^{13}$ reported statistically significant improvements in COP velocity in both AP and ML directions with eyes closed immediately following plantar massage. To maintain balance, the body must integrate appropriate sensory information from visual, vestibular, and somatosensory systems. ${ }^{14}$ By stimulating somatosensory information, an individual with CAI may have an increased ability to adjust to perturbations occurring in either the mediolateral or anteroposterior directions following plantar massage. ${ }^{15}$ LeClaire and Wikstrom $^{9}$ and McKeon and Wikstrom ${ }^{13}$ 
reported different conclusions when individuals were required to close their eyes, indicating that there is no consensus on the effect of plantar massage when vision is occluded. Therefore, the added constraint created by the removal of visual input may offset the potential benefits of plantar massage. However, despite the differences in outcome measures examined and testing procedures performed, each study found an improvement in postural control outcomes following plantar massage treatment. Therefore, the current evidence suggests that including plantar massage is associated with improvements in static postural control, regardless of differences in treatment protocol and outcome measure, when treating patients with CAI compared with other interventions.

Although all 3 studies supported plantar massage as a way to improve static postural control, each study utilized different elements of study and experimental design. Both Wikstrom et $\mathrm{al}^{12}$ and LeClaire and Wikstrom ${ }^{9}$ used crossover designs to evaluate several different massage interventions or techniques. Wikstrom et $\mathrm{al}^{12}$ focused exclusively on plantar massage using 3 different methods: manual massage, a patient-delivered ball massager, and a patientdelivered sensory brush. All treatments were 5 minutes in duration with static and dynamic postural control measures assessed at the beginning and end of each session. LeClaire and Wikstrom ${ }^{9}$ compared static postural control with eyes open and eyes closed following 5 minutes of clinician-delivered plantar massage or calf massage. Each study had washout periods between treatment sessions to mitigate the chance for carryover effect. McKeon and Wikstrom ${ }^{13}$ compared the effects of different sensorimotor interventions on single-limb balance while on a force plate. Participants were randomly assigned to 4 different treatment groups: control, joint mobilization, plantar massage, or calf stretching. Participants received six 5-minute treatments over a 2 -week period. Participants completed static postural control testing immediately after the first session of treatment and at the completion of all 6 treatments. Although McKeon and Wikstrom ${ }^{13}$ may be able to draw a stronger association between plantar massage and improved balance outcomes, as each patient received multiple treatment sessions, they only identified statistical significance immediately following the first treatment but not following 2 weeks of the intervention. Although each study investigated plantar massage in a unique way, all came to the same conclusion that plantar massage improves static postural control immediately following treatment when comparing preintervention and postintervention postural control.

Clinically, the critical appraisal of these studies demonstrates that plantar massage may be beneficial as an intervention during the rehabilitation of patients with CAI. ${ }^{9,12,13}$ Plantar massage is an intervention that does not require extra equipment and, therefore, can be easily integrated into a rehabilitation progression for patients following an ankle sprain to improve balance. Although the underlying mechanism to the development of CAI is unknown, it is apparent that minimizing static postural control deficits after an ankle sprain through rehabilitation is an important step in reducing the risk of long-term consequences. ${ }^{16}$ Multiple investigations have found reduced reinjury rates following the completion of balance and other sensorimotor training programs. ${ }^{17,18}$ It is also known that individuals who receive rehabilitation following an acute ankle sprain have better self-reported function. ${ }^{19}$ Although the outcomes and methods used in each study design are slightly different, all 3 investigations provided quality evidence that indicated that plantar massage acutely improved static balance in those with CAI. Therefore, for the purpose of this CAT, there is sufficient evidence to support the use of plantar massage to create immediate improvements in static postural control in patients with CAI.
This CAT is not without limitations. Wikstrom et $\mathrm{al}^{12}$ and LeClaire and Wikstrom ${ }^{9}$ only investigated the immediate effects of plantar massage and other interventions on balance, whereas McKeon and Wikstrom ${ }^{13}$ examined balance after a single treatment as well as after several treatment sessions. Each study also had differing testing procedures. In addition to this, there was one consistent author for all 3 studies, which may limit the generalizability of the findings in this CAT.

Future research should examine postural control measures following plantar massage intervention with both a CAI group and a control group. This would improve the quality of evidence and, therefore, may strengthen the level of recommendation. Future research should also focus on determining long-term effects of plantar massage on patients with CAI and incorporating it with other sensorimotor interventions. Ankle instability is not a condition that can be rehabilitated in one treatment session. Therefore, research should expand in ways that are more applicable to clinical practice, allowing clinicians to provide patients with the best outcomes possible. This could include more research examining the effect of postural control on dynamic balance. Finally, future research should also investigate postural control outcomes in acute ankle sprain patients who receive sensorimotor-directed rehabilitation immediately following their injury. Examining these ankle sprain patients long-term could help identify whether sensorimotor rehabilitation could potentially mitigate the balance deficits seen in patients with CAI. The clinical bottom line of this CAT was based on 3 moderate- to high-quality studies: 1 randomized controlled trial and 2 crossover studies. This recommendation should be revisited if further evidence becomes available within the area of this clinical question.

\section{Acknowledgments}

No financial support was used for this investigation. The authors have no financial affiliations (including research funding) or investment with any commercial organization that has a direct financial interest in any matter included in this manuscript. In addition, the authors have no other conflict of interest.

\section{References}

1. Gerber JP, Williams GN, Scoville CR, Arciero RA, Taylor DC. Persistent disability associated with ankle sprains: a prospective examination of an athletic population. Foot Ankle Int. 1998;19(10):653-660. PubMed ID: 9801078 doi:10.1177/107110079801901002

2. van Rijn RM, van Os AG, Bernsen RM, Luijsterburg PA, Koes BW, Bierma-Zeinstra SM. What is the clinical course of acute ankle sprains? A systematic literature review. Am J Med. 2008;121(4):324-331.e326. PubMed ID: 18374692 doi:10.1016/j.amjmed.2007.11.018

3. Hertel J. Functional anatomy, pathomechanics, and pathophysiology of lateral ankle instability. J Athl Train. 2002;37(4):364-375. PubMed ID: 12937557

4. Gribble PA, Delahunt E, Bleakley CM, et al. Selection criteria for patients with chronic ankle instability in controlled research: a position statement of the International Ankle Consortium. J Athl Train. 2014;49(1):121-127. PubMed ID: 24377963 doi:10.4085/ 1062-6050-49.1.14

5. Gribble PA, Bleakley CM, Caulfield BM, et al. Evidence review for the 2016 International Ankle Consortium consensus statement on the prevalence, impact and long-term consequences of lateral ankle sprains. Br J Sports Med. 2016;50(24):1496-1505. PubMed ID: 27259753 doi:10.1136/bjsports-2016-096189 
6. Hertel J, Olmsted-Kramer LC. Deficits in time-to-boundary measures of postural control with chronic ankle instability. Gait Posture. 2007;25(1):33-39. PubMed ID: 16446093 doi:10.1016/j.gaitpost. 2005.12.009

7. Wikstrom EA, Fournier KA, McKeon PO. Postural control differs between those with and without chronic ankle instability. Gait Posture. 2010;32(1):82-86. PubMed ID: 20418101 doi:10.1016/j. gaitpost.2010.03.015

8. Hoch MC, McKeon PO, Andreatta RD. Plantar vibrotactile detection deficits in adults with chronic ankle instability. Med Sci Sports Exerc. 2012;44(4):666-672. PubMed ID: 21959910 doi:10.1249/MSS. 0b013e3182390212

9. LeClaire JE, Wikstrom EA. Massage for postural control in individuals with chronic ankle instability. Athl Train Sports Health Care. 2012;4(5):213-219.

10. de Morton NA. The PEDro scale is a valid measure of the methodological quality of clinical trials: a demographic study. Aust J Physiother. 2009;55(2):129-133. PubMed ID: 19463084 doi:10.1016/ s0004-9514(09)70043-1

11. Powden CJ, Hoch JM, Hoch MC. Rehabilitation and improvement of health-related quality-of-life detriments in individuals with chronic ankle instability: a meta-analysis. J Athl Train. 2017;52(8):753-765. PubMed ID: 28704635 doi:10.4085/1062-6050-52.5.01

12. Wikstrom EA, Song K, Lea A, Brown N. Comparative effectiveness of plantar-massage techniques on postural control in those with chronic ankle instability. J Athl Train. 2017;52(7):629-635. PubMed ID: 28535097 doi:10.4085/1062-6050.52.4.02
13. McKeon PO, Wikstrom EA. The effect of sensory-targeted ankle rehabilitation strategies on single-leg center of pressure elements in those with chronic ankle instability: a randomized clinical trial. $J \mathrm{Sci}$ Med Sport. 2018;22(3):288-293. PubMed ID: 30244979 doi:10. 1016/j.jsams.2018.08.017

14. Winter DA, Patla AE, Frank JS. Assessment of balance control in humans. Med Prog Technol. 1990;16(1-2):31-51. PubMed ID: 2138696

15. McKeon PO, Stein AJ, Ingersoll CD, Hertel J. Altered plantarreceptor stimulation impairs postural control in those with chronic ankle instability. J Sport Rehabil. 2012;21(1):1-6. PubMed ID: 22318247 doi:10.1123/jsr.21.1.1

16. Miklovic TM, Donovan L, Protzuk OA, Kang MS, Feger MA. Acute lateral ankle sprain to chronic ankle instability: a pathway of dysfunction. Phys Sportsmed. 2018;46(1):116-122. PubMed ID: 29171312 doi:10.1080/00913847.2018.1409604

17. Emery CA, Rose MS, McAllister JR, Meeuwisse WH. A prevention strategy to reduce the incidence of injury in high school basketball: a cluster randomized controlled trial. Clin J Sport Med. 2007;17(1):1724. PubMed ID: 17304001 doi:10.1097/JSM.0b013e31802e9c05

18. Holme E, Magnusson SP, Becher K, Bieler T, Aagaard P, Kjaer M. The effect of supervised rehabilitation on strength, postural sway, position sense and re-injury risk after acute ankle ligament sprain. Scand J Med Sci Sports. 1999;9(2):104-109. PubMed ID: 10220845 doi:10.1111/j.1600-0838.1999.tb00217.x

19. Hubbard-Turner T. Lack of medical treatment from a medical professional after an ankle sprain. J Athl Train. 2019;54(6):671-675. PubMed ID: 31116568 doi:10.4085/1062-6050-428-17 